\title{
Genome-wide analysis of light-regulated alternative splicing mediated by photoreceptors in Physcomitrella patens
}

\author{
Hshin-Ping Wu', Yi-shin Su${ }^{\dagger}$, Hsiu-Chen Chen, Yu-Rong Chen, Chia-Chen Wu, Wen-Dar Lin and Shih-Long Tu*
}

\begin{abstract}
Background: Light is one of the most important factors regulating plant growth and development. Light-sensing photoreceptors tightly regulate gene expression to control photomorphogenic responses. Although many levels of gene expression are modulated by photoreceptors, regulation at the mRNA splicing step remains unclear.

Results: We performed high-throughput mRNA sequencing to analyze light-responsive changes in alternative splicing in the moss Physcomitrella patens, and found that a large number of alternative splicing events were induced by light in the moss protonema. Light-responsive intron retention preferentially occurred in transcripts involved in photosynthesis and translation. Many of the alternatively spliced transcripts were expressed from genes with a function relating to splicing or light signaling, suggesting a potential impact on pre-mRNA splicing and photomorphogenic gene regulation in response to light. Moreover, most light-regulated intron retention was induced immediately upon light exposure, while motif analysis identified a repetitive GAA motif that may function as an exonic regulatory cis element in light-mediated alternative splicing. Further analysis in gene-disrupted mutants was consistent with a function for multiple red-light photoreceptors in the upstream regulation of light-responsive alternative splicing.

Conclusions: Our results indicate that intensive alternative splicing occurs in non-vascular plants and that, during photomorphogenesis, light regulates alternative splicing with transcript selectivity. We further suggest that alternative splicing is rapidly fine-tuned by light to modulate gene expression and reorganize metabolic processes, and that pre-mRNA cis elements are involved in photoreceptor-mediated splicing regulation.
\end{abstract}

\section{Background}

Alternative splicing (AS) is a widespread mechanism in eukaryotes that generates two or more mRNAs from the same precursor mRNA (pre-mRNA) by using different splice sites. The mRNA splicing process is executed by the spliceosome, which consists of RNAs and more than 180 protein subunits. Intron recognition by spliceosomes is directed by intron-defining cis elements, including consensus $5^{\prime}$ and $3^{\prime}$ splice sites, branchpoint, and polypyrimidine tract [1]. Many splice sites are bound by spliceosomes for constitutive splicing, but others are selectively recognized for AS. Selectivity of alternative splice sites is influenced by regulatory cis elements

\footnotetext{
*Correspondence: tsl@gate.sinica.edu.tw

${ }^{\dagger}$ Equal contributors

Institute of Plant and Microbial Biology, Academia Sinica, Taipei 11529, Taiwan
}

(c) 2014 Wu et al.; licensee BioMed Central Ltd. This is an open access article distributed under the terms of the Creative Commons Attribution License (http://creativecommons.org/licenses/by/2.0), which permits unrestricted use, distribution, and reproduction in any medium, provided the original work is properly cited. enhancers or repressors. These sequences are recognized by trans-acting splicing regulators such as serine/arginine-rich (SR) proteins and heterogeneous nuclear ribonucleoproteins (hnRNPs) [2,3]. Binding of these proteins to splicing enhancers or repressors can promote or inhibit the recruitment of splicing machinery to alternative splice sites. The expression of splicing regulators is differentially regulated in different cell types, tissues, developmental stages, and environmental conditions. The presence of regulatory cis elements on pre-mRNA, dynamic binding of trans factors, differential expression, and post-translational modification of splicing regulators collectively determine the AS patterns of expressed genes and eventually generate tremendous transcriptome and proteome complexity [3,4]. Indeed, recent genomewide studies have shown that $42 \%$ to $61 \%$ of intron- 
containing genes in Arabidopsis and 33\% to 48\% of annotated genes in rice are alternatively spliced [5-7]. Many alternatively spliced transcripts contain premature termination codons (PTCs) that potentially leads to unproductive transcripts, truncated proteins or mRNA decay $[8,9]$. Therefore, AS also provides another level of gene regulation by modulating protein productivity, function, and transcript stability.

As sessile organisms, plants are constantly exposed to changes of environmental conditions. Light is one of the most crucial factors influencing plant growth and development. Fluctuation of light conditions affects the photosynthesis efficiency and rapidly stimulates developmental changes throughout the life cycle of plants. For example, when plant seedlings protrude from the soil (escape from etiolated state), developmental programs collectively called photomorphogenesis are rapidly turned on by light for vegetative growth. Plants have evolved sophisticated photoreceptor systems to regulate developmental processes of plant cells $[10,11]$. Plant photoreceptors include phytochromes, cryptochromes, phototropins, and ultraviolet (UV)-B photoreceptors. Phytochromes mainly perceive red and far-red light of the spectrum, whereas cryptochromes and phototropins perceive blue/UV-A light. Plant phytochromes can be further classified into two types, phytochrome B (phyB) and phytochrome A (phyA), according to their physiological responsiveness to red and far-red light, respectively [12]. Light perception by these photoreceptors triggers many biological processes, including gene regulation [11]. Global gene expression is rapidly altered by signal transduction or nuclear localization of lightactivated photoreceptors in response to light changes.

Accumulating data suggest that light regulation can occur at different stages of gene expression to control the abundance of functional gene products. Chromatin modification has been found tightly regulated by light $[13,14]$. A variety of chromatin modifications on lightresponsive gene loci have been identified. Such light-regulated dynamic alternations affect chromatin structure and play important roles in regulating transcriptional activity. At the molecular level, several light signaling components were proposed to function in chromatin regulation. A nuclear protein De-etiolated 1 (DET1) was shown to interact with UV-Damaged DNA Binding Protein 1 (DDB1) to regulate gene expression via chromatin binding [15]. In addition to chromatin regulation, light-regulated gene expression largely relies on the actions of light-signaling transcription factors (TFs), which have been extensively studied in the past few decades [16]. Although many TFs are involved in light signaling downstream of photoreceptors, the basic helix-loop-helix TF phytochrome interacting factors (PIFs), the basic zipper (bZIP) TF Long Hypocotyl 5
(HY5) and HY5 homolog (HYH) play a central role in integrating light signals to regulate light-responsive mRNA levels [17-19]. Light also regulates gene expression through translational control. Transcripts of photosynthetic genes are the target of light-mediated translational control [20-23]. More recently, a global survey of transcripts under translational regulation during photomorphogenesis in Arabidopsis has been reported [24]. Furthermore, light post-translationally affects abundance of key regulators through ubiquitin-dependent protein degradation. The CONSTITUTIVE PHOTOMORPHOGENIC 1 (COP1), an E3 ubiquitin ligase, target key components in light signaling for proteolysis via $26 \mathrm{~S}$ proteasome pathway [25]. The COP9 signalsome $(\mathrm{CSN})$, a protein complex with eight subunits in ubiquitin-proteasome pathway, is also involved in proteolysis of photomorphogenesis regulators in plants [26]. Although light regulation is found in almost every stage of gene expression, information on light regulation in the mRNA splicing step is relatively lacking.

In plants, many gene transcripts undergo AS in response to abiotic and biotic stresses [27]. Several reports have indicated that the abundance of alternatively spliced transcripts is altered by changing light conditions [28-30]. In addition, alternatively spliced transcripts of light signaling genes can function differently in light responses. Overexpression of an alternative isoforms of PIF6 in Arabidopsis was shown to elevate the efficiency of seed germination [31]. An alternatively-spliced isoform of COP1 also negatively regulate COP1 function in photomorphogenesis [32]. These results reveal that AS of light signaling genes can be important in regulating light responses. Most notably, a recent report identified an Arabidopsis mutant, reduced red-light responses in cry1cry2 background 1 ( $r r c 1)$, with impaired phyBdependent photomorphogenic responses [33]. RRC1 encodes a potential splicing factor with a C-terminal arginine/serine-rich (RS) domain that is important for phyB signaling. Further analysis in this report showed that AS patterns of SR protein gene transcripts are altered by red light and this effect is reduced in phyB and $r r c 1$ mutants. These data suggest that photomorphogenic responses in plants can be controlled by gene regulation at the mRNA splicing step.

Although these reports indicate that AS of some genes can be affected by light in plants, genome-wide analysis of AS in light responses is still not available. In this study, we used high-throughput mRNA sequencing (RNA-seq) to detect transcriptome changes during light exposure for 1 or $4 \mathrm{~h}$ of red or blue light. We examined the responsiveness of AS at red and blue light wavelengths to investigate the possible involvement of red and blue light-dependent mechanisms for splicing regulation. A simple model system, the moss Physcomitrella 
patens, was adopted for this approach. In general we found around $50 \%$ of moss genes undergoing AS in the simple-cell-type protonema. Intron retention (IR) is the most prevalent form of AS in Physcomitrella. Lightregulated AS events were identified with a statistic method and experimentally validated. Analysis of the top 1,000 light-regulated IR events showed that IR of most genes were rapidly induced within $1 \mathrm{~h}$, especially for genes functioning in chloroplast and translation. Furthermore, AS of many splicing-related and light signaling gene transcripts were also regulated by light. Comparison of IR pattern for wild type and a phytochrome-deficient mutant reveal the involvement of photoreceptors in immediate regulation of AS. Further analysis in gene-disrupted phytochrome mutants indicates that the phyB type of moss phytochromes is responsible for splicing regulation under red light. Altogether, our results suggest that light-regulated AS is important in shaping transcriptome for light responses. The splicing machinery is rapidly responsive to light. Photoreceptors are activated by specific wavelength of light to regulate AS, which results in the production of functional proteins for gene regulation and cellular processes during photomorphogenesis.

\section{Results}

\section{RNA-seq identified a substantial number of reads} supporting AS in Physcomitrella

In a previous study, we reported transcriptome changes in Physcomitrella in response to 1-h red light [34]. To further understand the light responsiveness in nonvascular plants, we expanded our approach with longer red-light exposure and with blue-light wavelength. Two-week-old Physcomitrella protonema were grown in the dark for 3 days (dark-grown control, D), then exposed to constant red (Rc) or blue light (Bc) for 1 (R1 and B1) and $4 \mathrm{~h}$ (R4 and B4). The expression of lightresponsive marker genes was first checked by RT-PCR (see Additional file 1: Figure S1). For RNA-seq, total RNA pooled from three biological replicates for each sample were subjected to cDNA library preparation (see Additional file 1: Figure S2). Sequencing involved the Illumina Hiseq 2000 platform with the paired-end (100-nt read length) method. After data trimming and filtering, nearly 195.5 million reads were generated (see Additional file 1: Table S1). Sequence reads were mapped to the Physcomitrella genome annotation V1.6 [35]. Approximately 138.4 million reads $(70.79 \%$ of total reads) were perfectly aligned to the reference genome, and $91 \%$ matched annotated gene regions. Reads mapped to the annotated gene regions were counted to calculate the reads per kilobase of exon model per million mapped reads (RPKM) for estimating gene expression [36].
Among the mapped reads, we found nearly 4.3 and 4.9 million reads located in the annotated intronic regions and across the exon-intron junctions, respectively. These reads were potentially generated from alternatively spliced transcripts in each sample. To further dissect these reads into different AS categories, we used the RACKJ package to isolate the reads supporting different AS events and statistically measure the light sensitivity of the event $[37,38]$. Reads aligned to AS sites for each sample were counted. Data from red- (R1 and R4) and blue-light (B1 and B4) samples were compared with the dark-grown control (D). To eliminate false-positive events, we filtered AS events originally detected by the following three criteria stepwise. In initially detected events from red- and blue-light samples, their corresponding genes with $<2$ reads mapped to exon regions were first removed; the remained events with $\geq 2$ reads aligned to splice junction(s) of AS sites were then retained; the events with at least 2 reads supported were then included in the final list. Chi-square test was used to compare read counts supporting and not supporting the AS event in each sample. For example, to calculate the significance of a red-light-regulated IR event, the number of intronic reads was compared with read numbers for corresponding gene exons (representing total transcripts) in D, R1, and R4 samples. Similarly, to calculate the significance of an exon skipping (ES) event, exon-skipped read counts supporting this event were compared with read counts aligned to the skipped exon. For the alternative donor site/alternative acceptor site (AltD/AltA) events, AltD/AltA read counts were then compared to constitutive donor and acceptor read counts. With this statistical method, the light sensitivity of an event could be quantified by normalization to the expression level of a corresponding gene or flanking exons.

\section{Frequencies of AS events in moss protonema are comparable to that in higher plants}

Although our RNA-seq data were mainly generated from light-treated samples, we believe the sequencing depth can reflect frequencies of AS events occurring in Physcomitrella. We detected totally 146,036 and 139,711 AS events in red- and blue-light samples, respectively (Figure 1A). Nearly 50\% of Physcomitrella genes were alternatively spliced in the protonema stage. Five major types of AS events were found: IR, ES, AltD, AltA, and AltD/AltA (AltDA) (Figure 1A and see Additional files 2 and 3 ). The number of events identified from red- and blue-light samples was surprisingly close. We compared all IR events identified in red- $(70,776)$ and blue$(69,722)$ light samples. Nearly $90 \%$ of IR events in both samples overlapped (Figure 1B). The data not only reveal the consistency of RNA-seq experiments, but also 


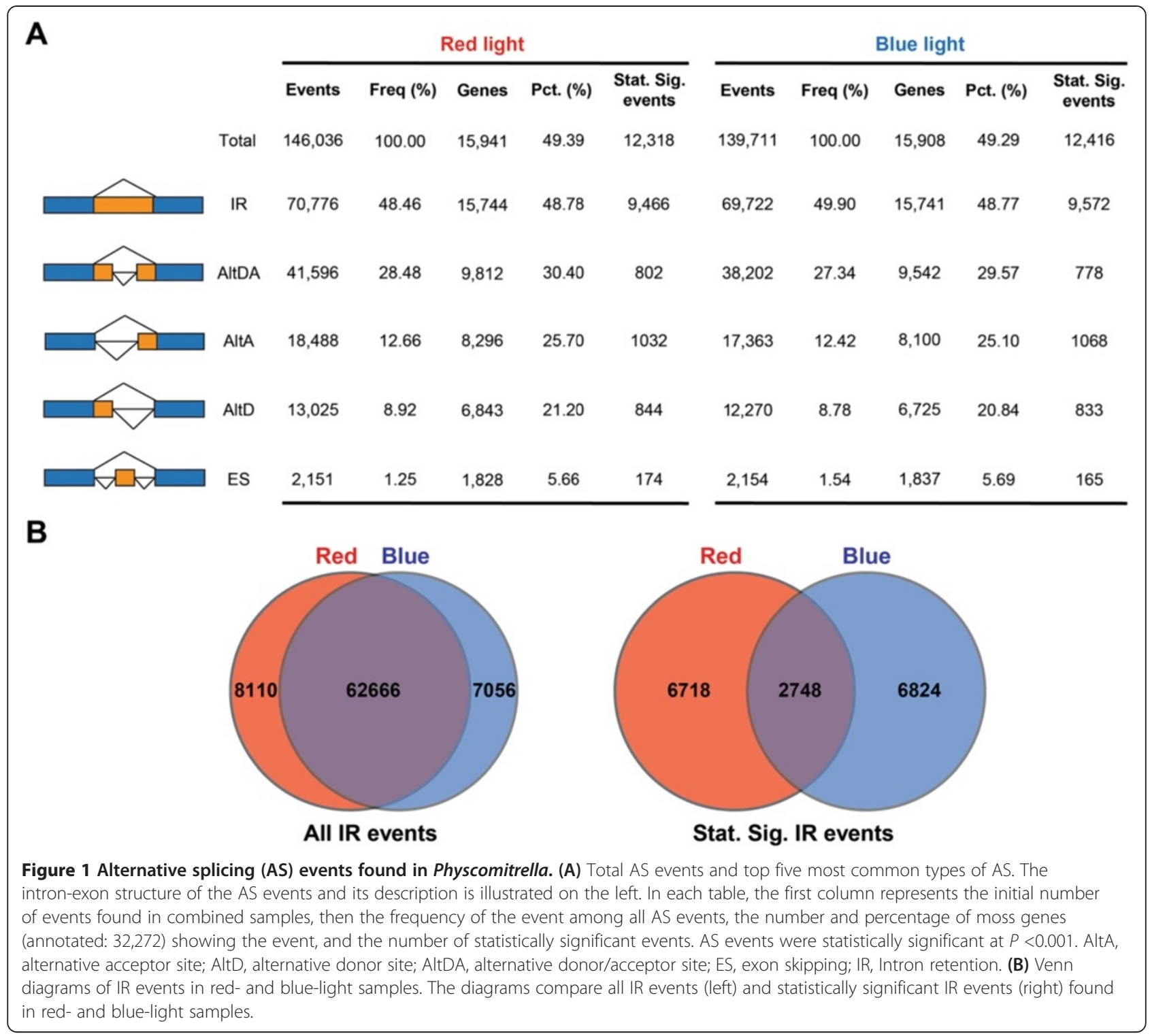

suggest that our AS survey covered the majority of AS events exist in Physcomitrella. However, when we compared the IR events with statistical significance in response to red $(9,466)$ and blue $(9,572)$ light, only $<30 \%$ of events were found responsive to both conditions. This result indicates that the events sensitive to light treatments are differentially regulated. A large portion of IR events is specifically responsive only to red or blue light.

In Physcomitrella, IR is the most common event, with a frequency of nearly $50 \%$ (Figure 1A). AltD and AltA are less abundant than IR, with higher usage of AltA than AltD. In addition, AltDA is more active than AltD and AltA. ES events identified in each light sample are only present in about $6 \%$ of moss genes. We conclude that the general pattern of AS is conserved among land plants. Complex and diverse AS events can be found in the simple-cell-type protonema of the ancient plant species.

\section{Light-responsive IR occurs on specific gene transcripts}

Similar to higher plants, transcription of moss genes are rapidly regulated in response to light changes [34]. AS may also play an important role in modulating the abundance of productive transcripts for light responses. Since IR is the most prevalent event responsive to the light, we first investigated the biological functions of light-responsive IR events. Because more than 9,000 IR events were statistically significant, we selected the top 1,000 events for subsequent analysis. Corresponding genes of the top 1,000 IR events responsive to Rc and $\mathrm{Bc}$ were subjected to functional enrichment analysis and classified according to Gene Ontology (GO) terms. 
Over-represented GO terms among functionally annotated genes are in Table 1. In both red- and blue-light data, translation-related terms were largely represented. Of these, genes encoding ribosomal proteins were highly enriched. In contrast, photosynthesis-related terms were enriched significantly in the top 1,000 Bc-responsive IR events, which implies that blue light immediately regulates IR of transcripts necessary for photosynthetic growth in the light. Our results strongly suggest that AS of transcripts involved in specific functions can be rapidly and differentially regulated by light.

The IR level of representative events for these GO terms was further validated by quantitative RT-PCR (qRT-PCR). We designed primer pairs to specifically detect the IR level and total transcripts of the corresponding gene. The IR level was normalized for total transcripts and then compared with data from the darkgrown control to generate the relative IR level. To calculate the IR level from RNA-seq data, we computed the intron reads per kilobase of retained intron per million mapped reads (IPKM) for each event. IPKM values were then normalized to RPKM values of corresponding genes and calculated for relative IR level compared to the dark-grown control. Indeed, IR levels for the selected genes were differentially regulated under both light conditions (Figure 2A and B). Patterns of light responsiveness were mostly comparable between qRT-PCR and RNA-seq data. This result supports the validity of our statistical method for identifying light-sensitive AS events.

\section{IR is rapidly induced by light in specific subset of chloroplast and ribosomal protein genes}

Data from qRT-PCR analysis for the top 1,000 lightresponsive IR events revealed an obvious light-sensitive pattern that most of IR events we validated showed immediate light induction at $1 \mathrm{~h}$ (Figure 2). To examine whether the pattern is common among light-responsive IR events, we compared IR levels (IPKMs) and corresponding gene expression levels (RPKMs) for the top 1,000 IR events. Similar to that observed by qRT-PCR data, more than $70 \%$ of the top 1,000 IR events were simultaneously upregulated within $1 \mathrm{~h}$ under both red and blue light, but their corresponding gene expression levels were overall unchanged (Figure 3A). This result demonstrates that light-activated IR is not associated with transcriptional activity. IR was induced immediately after exposure to light and became moderate in the later stage ( $4 \mathrm{~h}$ under light), which suggests that splicing of these introns was rapidly inhibited by light. Rapid IR induction was also found for chloroplast and ribosomal protein genes (Figure 3B and $\mathrm{C}$ ), which further reveals that splicing inhibition occurred in transcripts for specific biological processes.
Table 1 Over-represented Gene Ontology (GO) terms of top 1,000 intron retention (IR) genes regulated by red and blue light

\begin{tabular}{ll}
\hline \multicolumn{2}{c}{ Red light } \\
\hline GO term & $\boldsymbol{P}$ value \\
\hline MF: structural constituent of ribosome & $3.95 \mathrm{E}-29$ \\
CC: ribosome & $9.50 \mathrm{E}-25$ \\
BP: translation & $1.22 \mathrm{E}-22$ \\
CC: integral to membrane & $1.19 \mathrm{E}-06$ \\
BP: cell redox homeostasis & $1.96 \mathrm{E}-06$ \\
CC: mitochondrion & $8.95 \mathrm{E}-05$ \\
MF: glycerone kinase activity & $9.31 \mathrm{E}-05$ \\
\hline
\end{tabular}

A total of 1,000 genes were submitted to the GOBU functional enrichment tool, which resulted in 689 unique genes with annotations. Terms are ranked by $P$ value of over-representation and are included in list if $P<1 \mathrm{E}-04$. BP: Biological process; CC: Cellular component; MF: Molecular function.

\begin{tabular}{ll}
\hline \multicolumn{2}{c}{ Blue light } \\
\hline GO term & $\boldsymbol{P}$ value \\
\hline CC: chloroplast & $4.67 \mathrm{E}-33$ \\
MF: structural constituent of ribosome & $1.66 \mathrm{E}-29$ \\
CC: ribosome & $5.14 \mathrm{E}-27$ \\
BP: translation & $1.63 \mathrm{E}-20$ \\
BP: reductive pentose-phosphate cycle & $1.47 \mathrm{E}-16$ \\
CC: photosystem II & $2.36 \mathrm{E}-10$ \\
BP: photosynthesis, light harvesting & $6.62 \mathrm{E}-10$ \\
CC: photosystem I & $8.56 \mathrm{E}-10$ \\
CC: stromule & $2.32 \mathrm{E}-09$ \\
MF: chlorophyll binding & $2.53 \mathrm{E}-08$ \\
CC: thylakoid & $6.47 \mathrm{E}-08$ \\
BP: photorespiration & $1.00 \mathrm{E}-07$ \\
CC: chloroplast envelope & $1.12 \mathrm{E}-07$ \\
BP: photosynthesis & $3.92 \mathrm{E}-07$ \\
CC: oxygen evolving complex & $5.16 \mathrm{E}-07$ \\
CC: cytosolic ribosome & $3.22 \mathrm{E}-06$ \\
MF: rRNA binding & $1.68 \mathrm{E}-05$ \\
BP: cell redox homeostasis & $2.21 \mathrm{E}-05$ \\
BP: translational elongation & $2.26 \mathrm{E}-05$ \\
MF: ribulose-bisphosphate carboxylase activity & $2.86 \mathrm{E}-05$ \\
BP: nitrate assimilation & $5.10 \mathrm{E}-05$ \\
MF: glycerone kinase activity & $7.63 \mathrm{E}-05$ \\
\hline A tota of 1,00 gens wer submted to & \\
\hline
\end{tabular}

A total of 1,000 genes were submitted to the GOBU functional enrichment tool, which resulted in 733 unique genes with annotations. Terms are ranked by $P$ value of over-representation and are included in list if $P<1 \mathrm{E}-04$. $\mathrm{BP}$ : Biological process; CC: Cellular component; MF: Molecular function.

IR usually produces PTC and generates unproductive transcripts or truncated proteins. To further confirm that retained introns in IR transcripts generated PTCs, we computed the proportion of PTC-occurring IR 


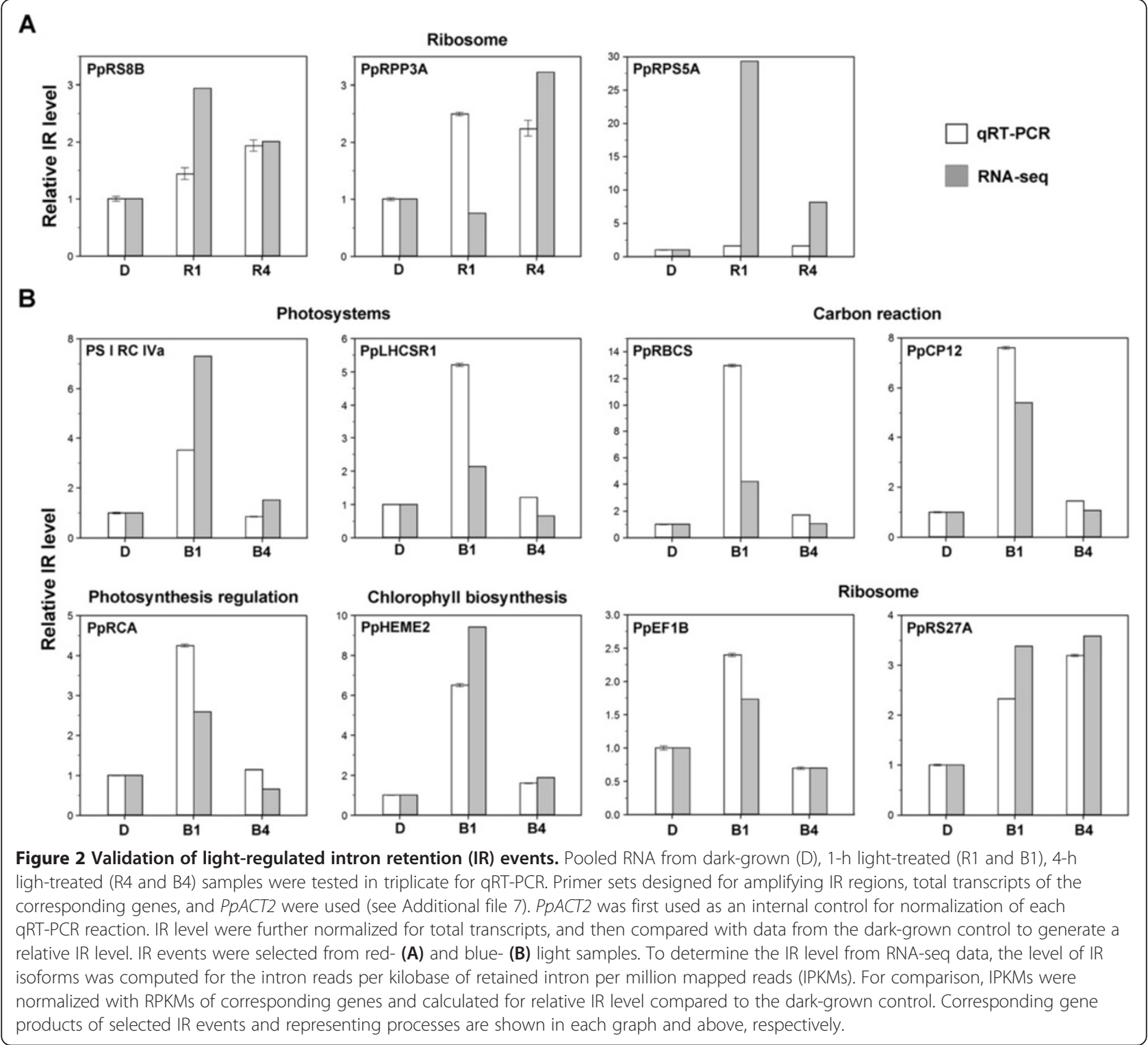

transcripts. We found most of IR transcripts had potential PTCs in the retained intron or downstream regions (see Additional file 1: Table S2). These results suggest that light repress intron splicing for certain gene transcripts may generate unproductive species to temporally attenuate specific biological responses after light exposure.

\section{IR of splicing factor gene transcripts were regulated by light}

Plant genomes contain a large amount of splicingrelated genes encoding core spliceosome components, splicing factors, and regulators [39]. Most of them are conserved among plants and metazoans. Many transcripts for these splicing-related proteins especially splicing regulators also show extensive AS patterns that are altered by environmental stresses $[39,40]$. Although splicing-related genes were not highly enriched in our data, those showing light-regulated AS events potentially impact on splicing regulation for light responses. We surveyed the lists of genes with IR events responsive to red and blue light and found more than 70 splicingrelated genes in red- and blue-light data, respectively. These genes can be categorized into several groups encoding SR proteins, KH-domain proteins, polypyrimidine tract binding proteins (PTBPs), DEAD box helicases, spliceosome components, and other splicing factors (see Additional files 4 and 5). We selected SR protein genes in the top 1,000 events of both red- and blue-light data for validation by qRT-PCR (Additional file 1: Figure S5). Interestingly, although majority of the top 1,000 IR events were rapidly induced by light, IR for all of these genes showed the pattern of light repression 


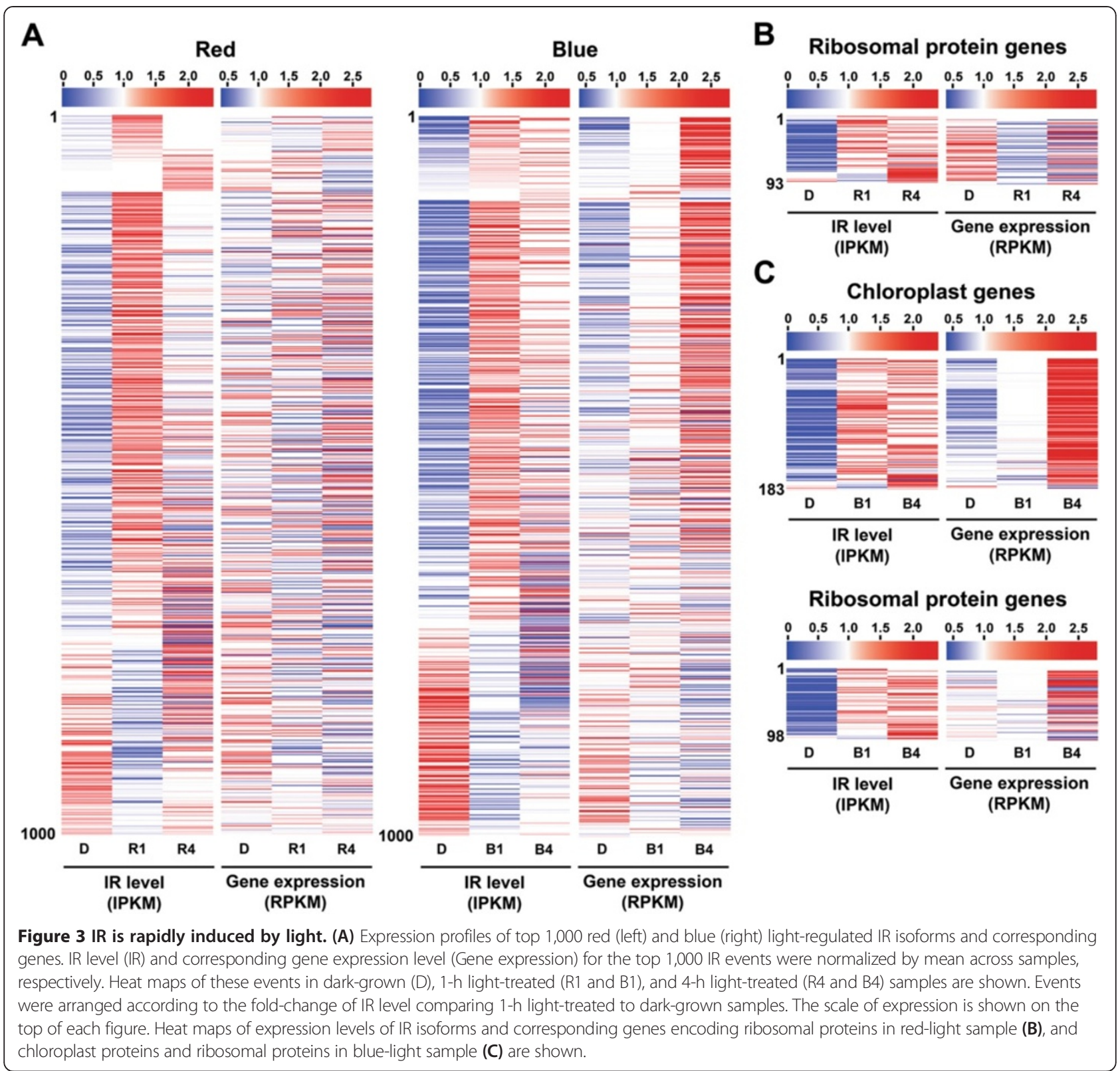

(Figure 4). These results suggests that light enhance splicing activity for these genes to generate productive transcripts, which allow to synthesize more functional SR proteins for subsequent splicing regulation under the light.

\section{Light signaling genes were alternatively spliced for photomorphogenic control}

In Arabidopsis, alternatively spliced isoforms of light signaling genes have been found involved in plant development [31,32]. However, whether AS of light signaling gene transcripts can be modulated is unknown. Light signaling genes in our AS data may play important roles in photomorphogenesis. After surveying potential light signaling genes in moss genome and those showing light-regulated AS in our data, we found 36 of them alternatively spliced under red or blue light (Table 2 and see Additional file 6). These genes cover almost all the steps of photomorphogenic gene regulation from chromatin remodeling to post-translational protein degradation. To our surprise, almost all members of several light signaling gene families including $H Y 5 / H Y H$, PIFs, COP1, CSNs, DET1, and DDB1 underwent lightregulated AS. AS regulation for these genes potentially modulate the function or abundance of light signaling factors, and could impact on both red- and blue-light signaling pathways.

We next analyzed the patterns of light-regulated AS for these genes. Since all 36 genes underwent light- 


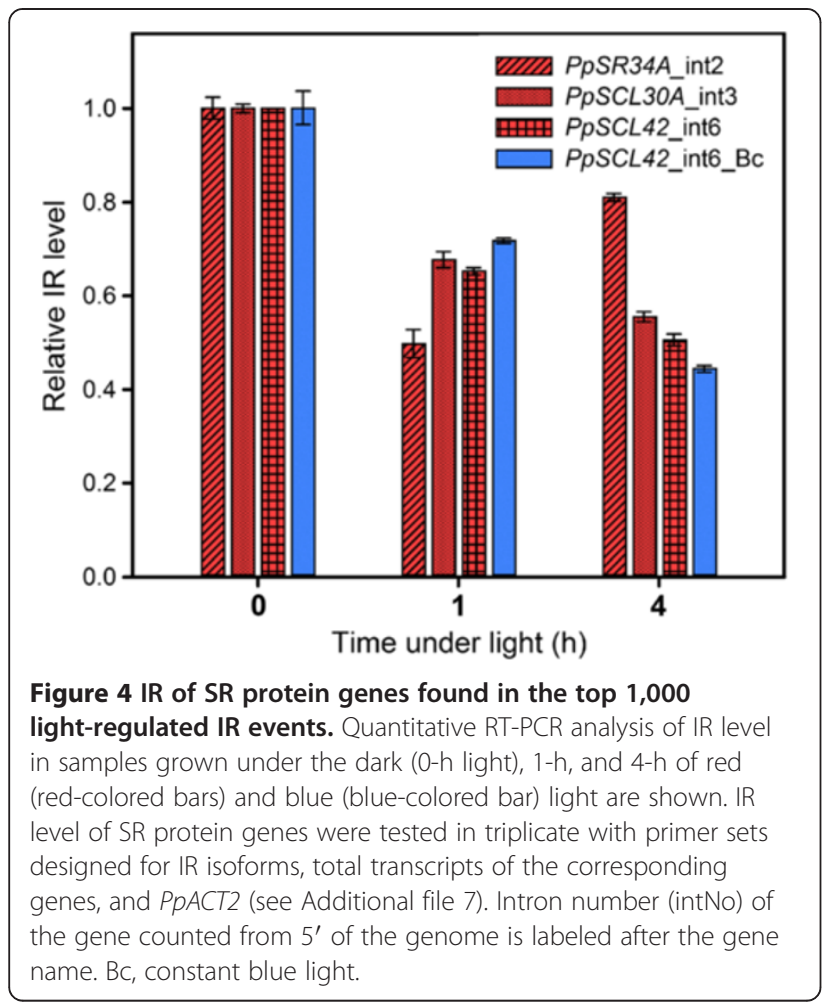

regulated IR, we therefore calculated IPKM and RPKM for the IR events occurred in these genes and validated by RT-PCR (Figure 5 and Additional file 1: Figure S6). Although IR patterns showed variations among these gene families, they can be divided into three groups. The first group of gene families including $H Y 5 / H Y H$, $C O P 1$, and CSNs, which encode key regulators in the downstream of both red- and blue-light signaling pathways, showed relatively consistent IR repression or induction after exposing $1 \mathrm{~h}$ of red and blue light (Figure 5A). Enhancing intron splicing in $H Y 5 / H Y H$

Table 2 Light signaling genes in Physcomitrella showed light-regulated AS

\begin{tabular}{llll}
\hline $\begin{array}{l}\text { Gene } \\
\text { families }\end{array}$ & $\begin{array}{l}\text { Annotated genes in } \\
\text { Physcomitrella }(\boldsymbol{n})\end{array}$ & $\begin{array}{l}\text { Genes alternatively spliced } \\
\text { under red or blue light }(\boldsymbol{n})\end{array}$ & $\begin{array}{l}\text { AS } \\
\text { types }\end{array}$ \\
\hline NPH3 & 26 & 7 & IR; AltA \\
HY5/ & 2 & 2 & IR; AltA; \\
HYH & & 4 & ES \\
PIFs & 4 & & IR; AltD; \\
& & 1 & AltA \\
BBX22 & 4 & 1 & IR \\
ELF3 & 3 & 6 & IR \\
COP1 & 9 & 10 & IR; AltA \\
CSN1-8 & 13 & 3 & IR; AltA \\
DET1 & 3 & & IR; AltD; \\
& & 2 & AltA \\
DDB1 & 2 & & IR \\
\hline
\end{tabular}

transcripts could allow the production of more functional $\mathrm{HY} 5 / \mathrm{HYH}$, the positive regulator, to enhance photomorphogenesis responses. Meanwhile, immediate inhibition of intron splicing by light for CSNs, the photomorphogenesis repressor, then may temporarily abolish degradation of light signaling factors [26]. The result in COP1 family is elusive because IR repression of COP1 transcripts potentially generates more E3 ligase for degrading light signaling regulators [25]. One explanation is COP1 may be required to degrade darkaccumulated photomorphogenesis repressors in the early stage of light exposure. It is also possible that COP1 in non-vascular plants have different functions.

The second group of transcripts from NPH3 and PIFs genes showed diverse patterns under their acting light conditions, that is, NPH3 under blue light and PIFs under red light, but rapid IR induction under inactive light conditions (Figure 5B). IR regulation in members of each gene family may promote differential activities of these factors under their acting wavelengths. DET1 and DDB1 represent the third group, which then showed diverse patterns under both red and blue lights (Figure 5C). In summary, our data indicate that light regulates AS of light signaling gene transcripts encoding central regulators downstream of both light signaling pathways as well as factors involved in specific light responses. The regulation could, at least partially, affect the abundance of light signaling factors and impact on the photomorphogenic control.

\section{HYH transcripts show complex but light-regulated AS in $5^{\prime}$ untranslated region}

Among light signaling genes with light-regulated AS, $H Y H$ showed unique AS pattern that IR was repressed by both red and blue light. HYH interacts with HY5 and converges red- and blue-light signaling pathways for regulating transcription of light-responsive genes [41]. AS regulation occurring in $H Y H$ transcripts could have a strong effect on photomorphogenic gene regulation. In Physcomitrella, HY5 and HYH are annotated as HY5 Homolog 1 and 2 (PpHYH1 and PpHYH2). According to our mapping result, Sanger sequencing of RT-PCR products, and previous version of annotation, we revised the exon-intron structure for the $\mathrm{PpHYH} 2$ gene. New gene models mainly encode 10 isoforms with complex IR and ES in the long 5' untranslated region (5'UTR) (Figure 6A and Additional file 1: Figure S4). All three introns in 5'UTR are retained individually. Most of the IR isoforms can be translated into a 167 amino-acid protein with a bZIP DNA binding domain (DBD) in the C-terminal half, except the retention of intron 3 (isoform 8 and 9) or skipping of exon 3 (isoform 10) results in an extended $\mathrm{N}$ terminus of $\mathrm{PpHYH} 2$ protein. Although based on the sequence prediction all of the alternatively spliced transcripts of $\mathrm{PpHYH} 2$ can produce a polypeptide with a bZIP DBD, IR in the 5 'UTR potentially 


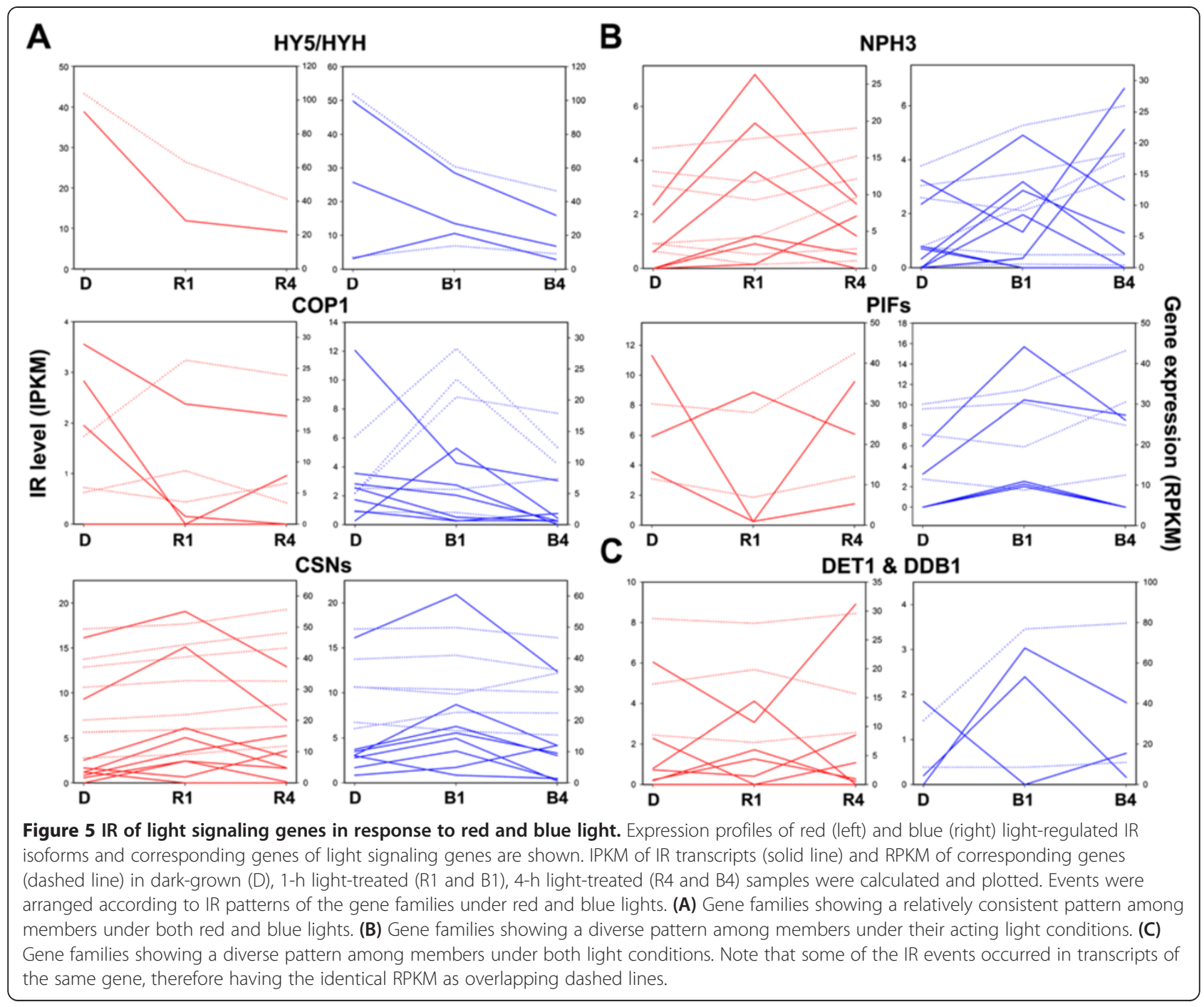

generates upstream open reading frames (uORFs). uORFs usually play a negative role in affecting translation of the downstream primary ORF [42]. IR in PpHYH2 5'UTR may increase the number of uORFs that could repress gene expression.

To validate the IR level of $\mathrm{PpHYH2}$ transcripts, we performed qRT-PCR. Primer pairs were designed to amplify the retained introns to determine IR level and constitutively-expression regions for total transcripts. Data showed that IR in PpHYH2 5'UTR were significantly repressed by red and blue light (Figure 6B). Blue light had a strong effect on the IR inhibition. This result suggests that intron splicing of $\mathrm{PpHYH2}$ transcripts is enhanced by light, which may shorten the PpHYH2 5' UTR and decrease the chance of uORF formation. This could increase the translation efficiency of the primary $\mathrm{ORF}$ and produce more functional $\mathrm{HYH}$ for positively regulating photomorphological gene transcription under the light.

\section{AltD and AltA are also regulated by light}

Besides IR, AltD and AltA are also frequently found in plants. In Physcomitrella, we also observed large quantity of AltDA, AltD, and AltA events significantly affected by light (Figure 1). We again performed GO analysis to identify over-represented functions for these events. Red and blue light-responsive AltDA, AltD, and AltA events were pooled for functional enrichment analysis (see Additional file 1: Table S3). GO terms overrepresented in red- and blue-light data were overall similar, except chloroplast-related terms were highly enriched from the blue light-regulated events. Of note, genes encoding nuclear proteins, kinases, and RNA polymerase II transcription factors or functioning in the ubiquitin-degradation catabolic process, RNA processing, and chromatin modification were enriched in the light-responsive AltD/AltA events. These gene products mostly act as regulators for gene expression, which implies that light may primarily and selectively modulate 


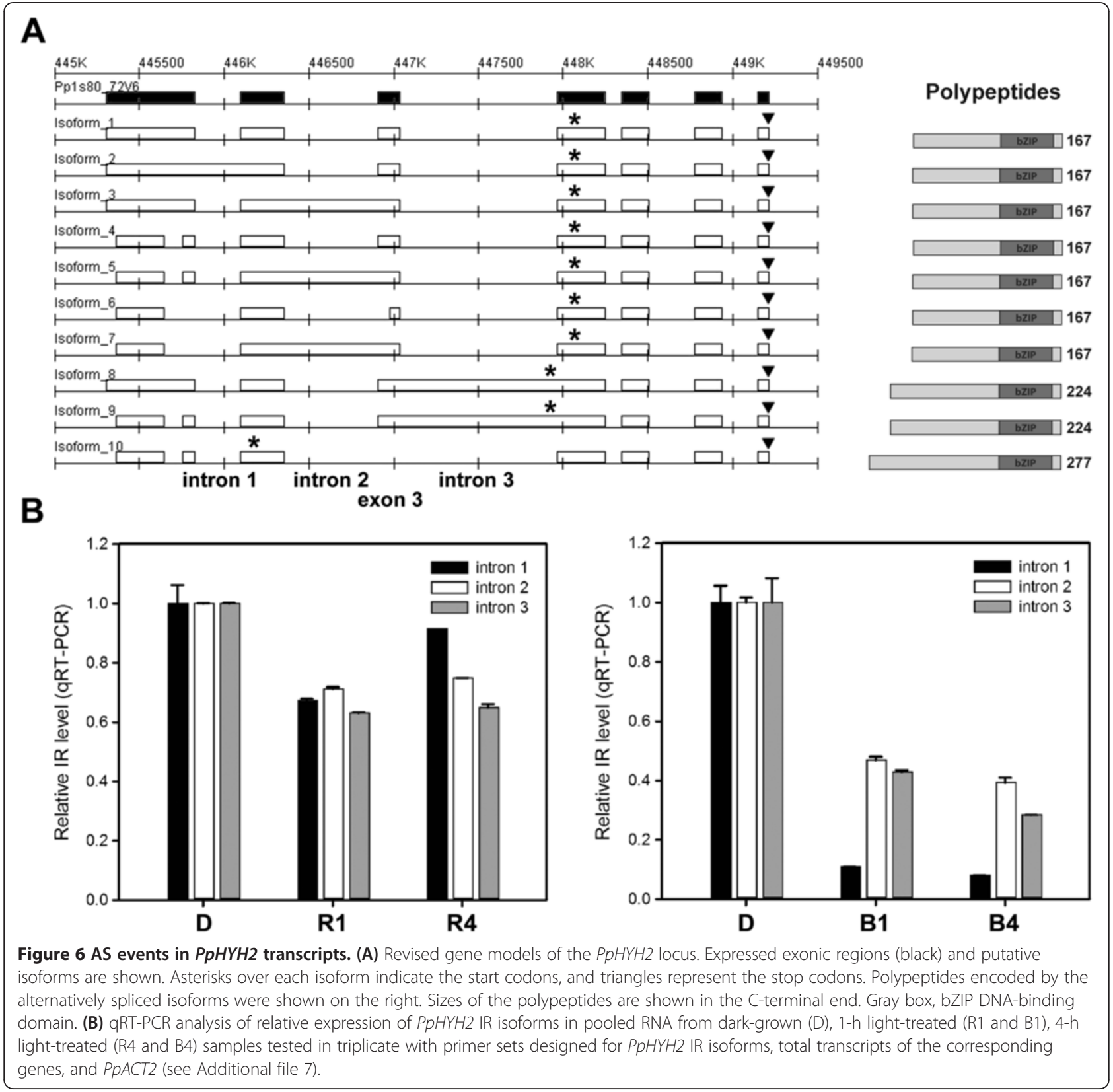

AS of key components involved in gene regulation. Expression of alternative transcripts in certain enriched GO terms was validated by high-resolution RT-PCR (see Additional file 1: Figure S4) [43]. In conclusion, our data reveal that light also regulates AltD and AltA in gene transcripts, especially for genes encoding regulatory factors in Physcomitrella.

\section{Motif analysis of IR regions reveals a repetitive GAA cis} element enriched in exons

Regulatory cis elements presence in the AS regions can serve as binding sites for trans splicing factors to recruit the splicing machinery. In our data, a substantial number of events was highly light-regulated, which indicates that specific cis and trans components may also participate in the AS regulation. To examine whether the AS regions of light-regulated transcripts share common cis elements, we analyzed the light-regulated IR events using the MEME suite for motif search [44]. Three datasets were generated for motif analysis: sample, reference, and control datasets. To obtain the sample dataset, we extracted 200 nt of upstream and downstream flanking sequences from both $5^{\prime}$ and and $3^{\prime}$ splice sites of the top 500 retained introns that were responsive to red and blue light, respectively (Figure 7A). For introns shorter than $200 \mathrm{nt}$, the whole intron and 
B
A
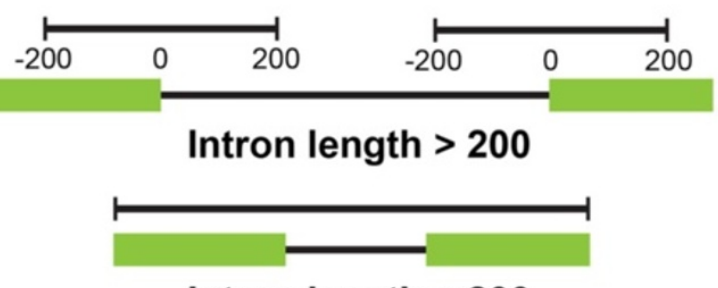

Intron length $<200$

C

C Donor side

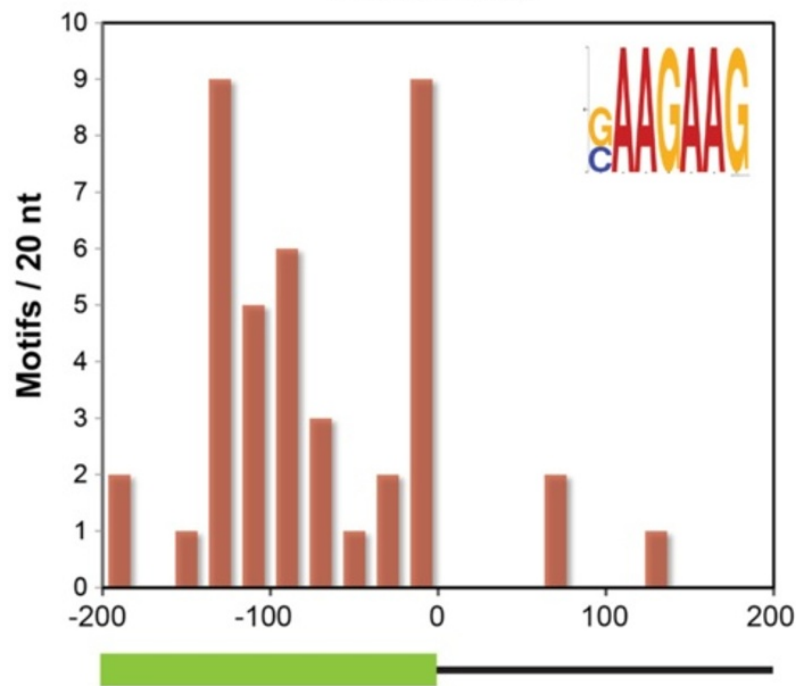

D

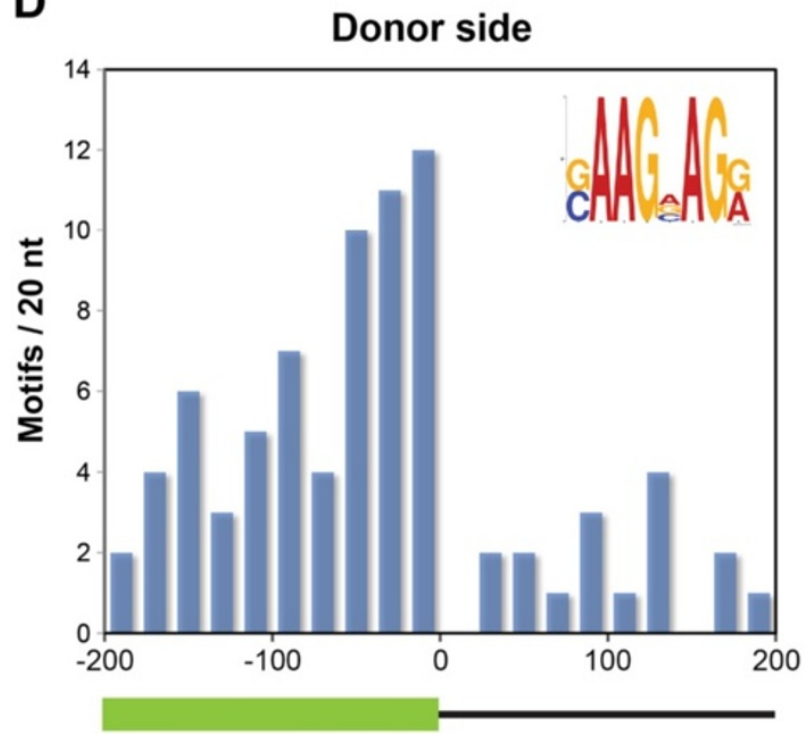

Red
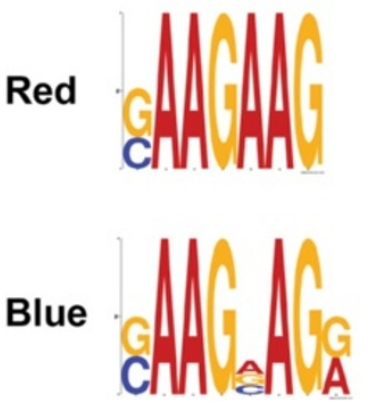

$E$-value $=1.9 \mathrm{E}-36$

$E$-value $=1.7 \mathrm{E}-38$
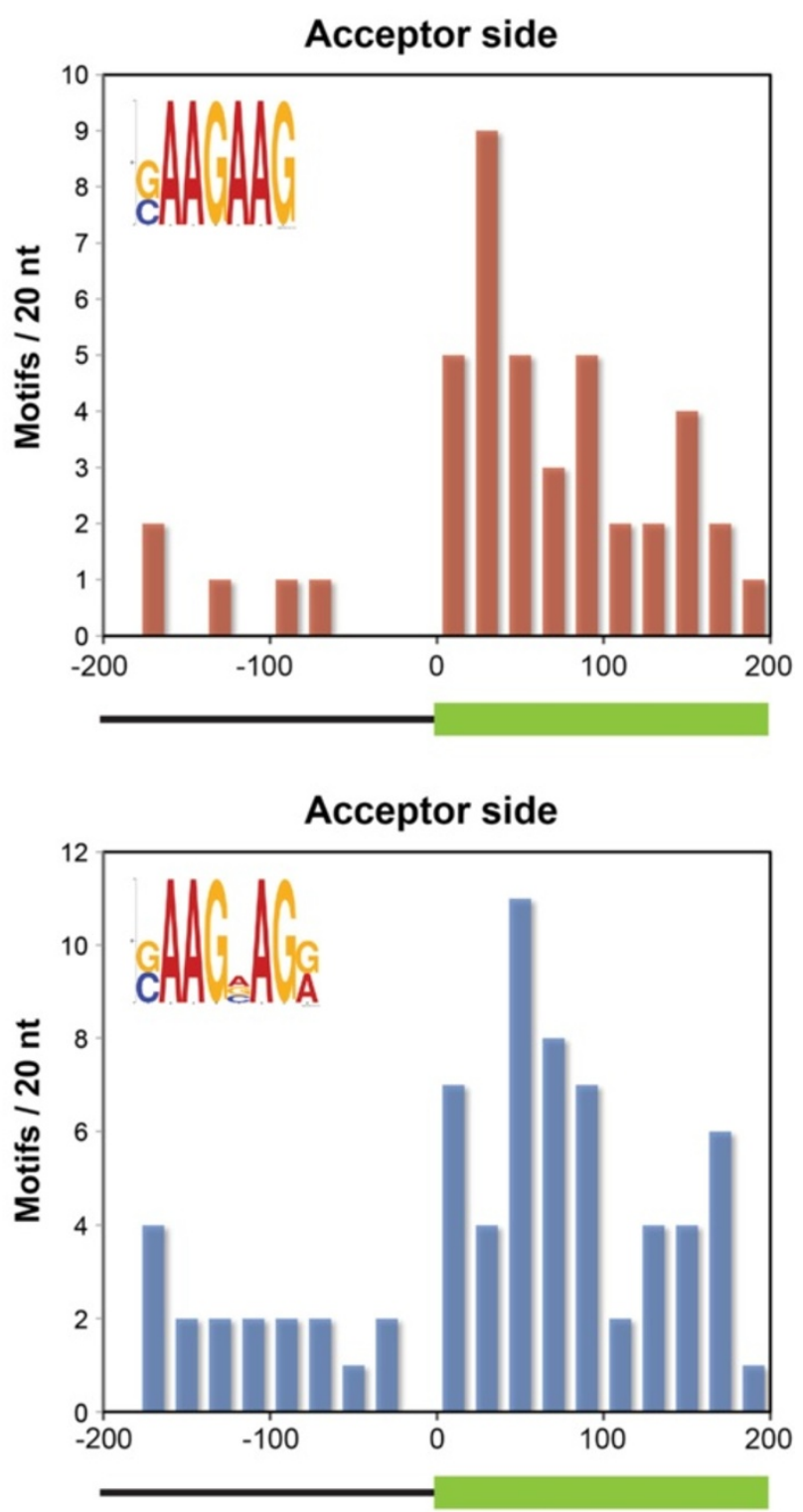

Figure 7 (See legend on next page.) 
(See figure on previous page.)

Figure 7 Regulatory cis element in light-regulated IR regions. (A) Illustration of sequence extraction for motif search. (B) The repetitive GAA motif enriched in red (top) and blue (bottom) light-regulated IR regions. The E-value indicating the statistical significance of the motif is shown on the right of the motif. Frequency distribution of the repetitive GAA motif in red (C) and blue (D) light-regulated IR regions are plotted. Frequencies of the motif were generated by counting the numbers of motif hits in windows of 20 nt near the donor and acceptor sites of light-regulated IR regions. Only input sequences extracted from donor and acceptor sites of the retained introns (longer than $200 \mathrm{nt}$ ) were used for motif counting. The green bar represents the exon and the solid line indicates the retained intron.

adjacent exons were included. To generate the reference dataset, sequences in sample dataset were randomly shuffled. For the control dataset, sequences were extracted similar to that for the sample dataset but from 500 randomly selected IR events that were not statistically significant. We searched for conserved motifs from 7 to 12 mers. To further confirm the identified motifs preferentially located in the light-regulated IR regions, we compared the occurrence of the motif in the sample dataset to that in the control dataset based on the enrichment level with a $P$ value of 0.001 by Fisher's exact test.

In the red-light data, motif search yielded a highly conserved 7-nt sequence, [GC]AAGAAG, over-represented in red light-regulated IR regions, with an $E$-value of $1.9 \mathrm{E}-36$ (Figure 7B, upper logo). Interestingly, the motif identified from blue-light-regulated IR regions, [GC]AAG[AGC]AG[GA], shared a similar pattern with the red-light motif. Although several nucleotides on this element are less conserved, it can be defined as a purine-rich motif with a repetitive GAA sequence. We also examined the distribution of the repetitive GAA motifs near donor and acceptor sites of retained introns. Frequency distribution of both red- and blue-light motifs were plotted by counting the number of motif hits in windows of $20 \mathrm{nt}$. Most of the motif hits were located in adjacent exons of the retained introns, in general showing a higher frequency close to the splice sites (Figure 7C and D). This evidence further supports that the repetitive GAA cis element is an exonic splicing regulator that functions in light-regulated IR. The similar motif pattern in both red- and blue-light data reveals that the repetitive GAA element may commonly function as a binding site for splicing regulators in red and blue lightmediated splicing regulation.

Phytochromes primarily participate in splicing regulation Photomorphogenic gene regulation mainly relies on light-sensing photoreceptors. Our data showed that AS in Physcomitrella is immediately affected within $1 \mathrm{~h}$ of red- and blue-light exposure. Such early response is likely mediated directly by photoreceptors. To determine whether photoreceptors are involved in the light regulation of AS, we compared IR pattern in wild type (WT) and a mutant that is defective in red light sensing. In a previous study, we generated a Physcomitrella knockout mutant with gene disruptions at loci encoding the phytochromobilin synthase (HY2) and phycourobilin synthase (PUBS). The pubs hy2 double mutant is defective in biosynthesis of phytochrome chromophore and characterized to be a phytochrome-deficient mutant [34]. RNA-seq data previously generated for the pubs hy 2 double mutant were further analyzed. In WT, more than $70 \%$ of the top 1,000 IR events significantly affected after 1-h red light showed the pattern of light induction (Figure 8A, left panel). However, retention of these introns was mostly decreased in the pubs hy 2 double mutant after $1 \mathrm{~h}$ of red light exposure. On the other hand, those top 1,000 IR events repressed by red light in WT (bottom part of the list) also became largely unresponsive in the phytochrome-deficient mutant. Corresponding gene expression level for the top 1,000 IR events showed that light-mediated changes of IR level are not associated with transcriptional activity (Figure 8A, right panel). Overall, IR was rapidly induced by light in WT but misregulated globally in the pubs hy2 double mutant, suggesting the involvement of phytochromes in splicing regulation.

Seven phytochromes are found in Physcomitrella and physiological functions for several of them have been characterized [45-48]. To further determine the role of individual phytochromes in regulation of AS, we generated knockout mutants for PpPHY1, PpPHY2, PpPHY3, and PpPHY4 by gene targeting and designated them as phy1, phy2, phy3, and phy4 (see Additional file 1: Figure S7). IR level of two genes encoding the ribosomal protein PpRPP3A and SR protein PpSCL42 were measured by qRT-PCR in WT and four phytochrome knockout mutants grown under the dark, $1 \mathrm{~h}$, and $4 \mathrm{~h}$ of red light (Figure 8B and Additional file 1: Figure S5). Among four phytochrome-knockout mutants, we found phy1 and phy3 mutants still behave similarly as the WT with increased IR level for PpRPP3A and decreased for PpSCL42. However, IR of both genes was less responsive to red light in phy2 and phy4 mutants. Interestingly, PpPHY1 and PpPHY3 have been phylogenetically clustered and physiologically characterized as the phyA-type members. The rest of Physcomitrella phytochromes belong to the phyB type $[48,49]$. Our results suggest phyB-type phytochromes play more dominated roles under red light condition for IR regulation. Because the single mutants still showed partial light responsiveness, we propose that multiple phyB-type phytochromes are 


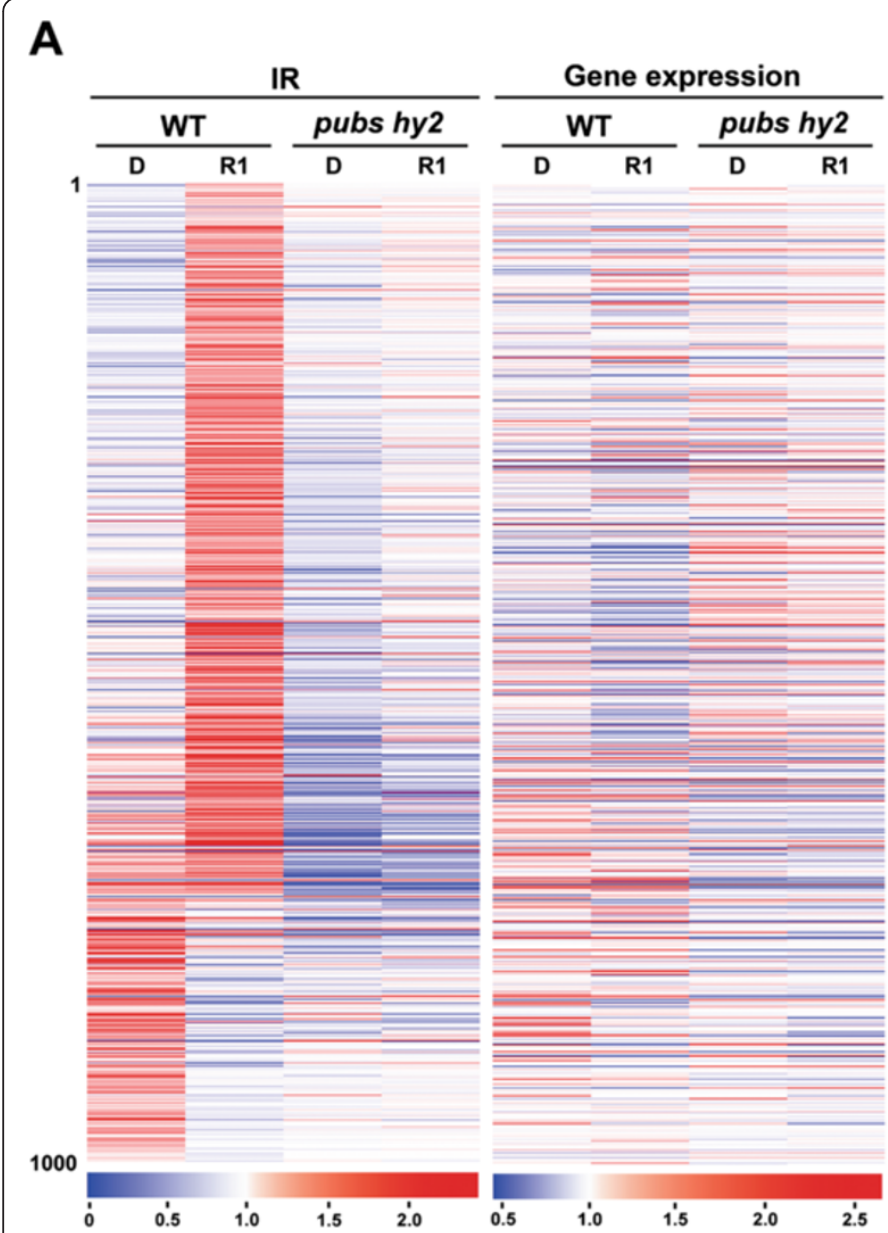

B
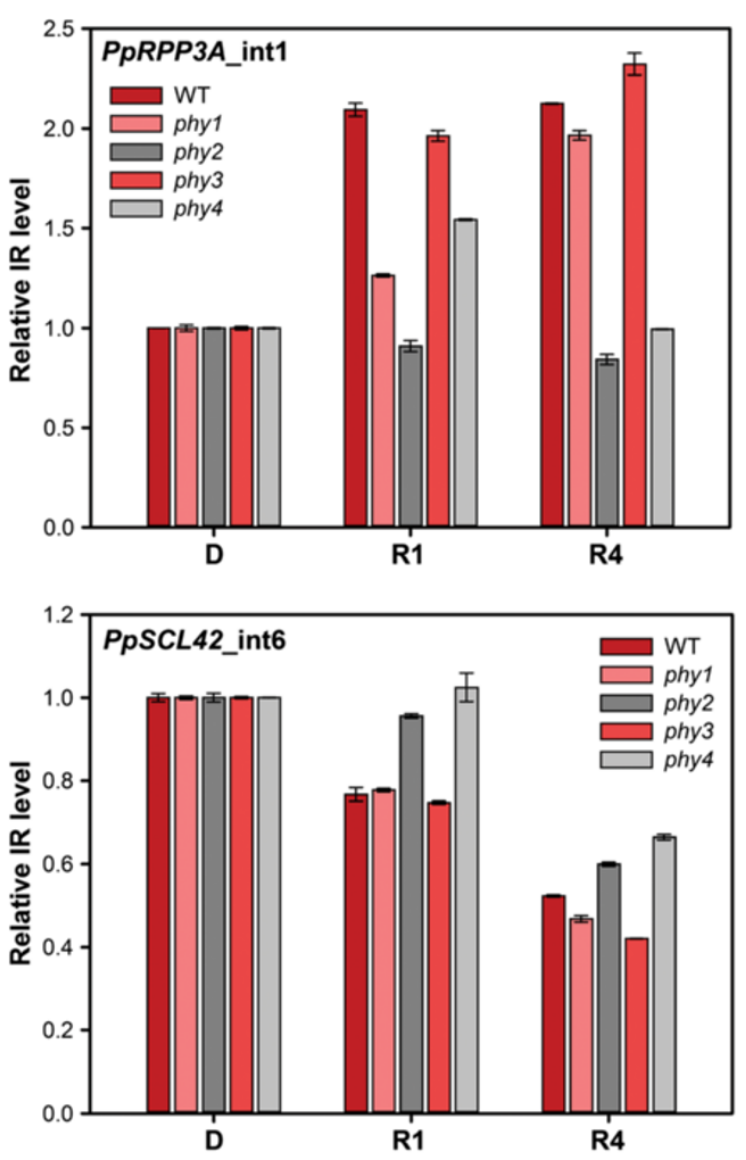

Figure 8 Red light induction of IR is misregulated in the phytochrome-deficient and -knockout mutants. (A) Expression profiles of top 1,000 red light-regulated IR isoforms and total transcripts of corresponding genes in wild type (WT) and pubs hy2 mutant are shown. IR level (IR) and corresponding gene expression level (Gene expression) for the top 1,000 IR events were normalized by mean across WT and mutant samples. Heat maps of these events in dark-grown (D) and 1-h red light-treated (R1) samples are generated. Events were arranged according to the fold-change of IR level comparing 1-h red light-treated to dark-grown samples in WT. The scale of expression is shown in the bottom. (B) IR of PPRPPP3A and PpSCL42 in WT and phytochrome-knockout mutants. Quantitative RT-PCR analysis of IR level in samples from WT and four phytochrome-knockout mutants (phy1, phy2, phy3, phy4) grown under dark (D), 1-h (R1), and 4-h (R4) of red light. Both genes were tested in triplicate with primer sets designed for IR isoforms, total transcripts of the corresponding genes, and PpACT2 (see Additional file 7).

involved in the regulatory process. In conclusion, the photoreceptors, at least phytochromes, are the determinants for regulating AS.

\section{Discussion}

AS is another level of gene regulation for light responses in plants

Regulation of gene expression can occur at different levels to control the abundance of functional gene products for cellular differentiation and morphogenesis. In plant photomorphogenesis, regulation at the chromatin, transcription, translation, and post-translational levels have been reported and well characterized [13,14,17-19,24-26]. In this study, we performed a transcriptome-wide study to suggest that global gene regulation during light exposure also takes place at the
mRNA splicing step. By taking advantage of nextgeneration sequencing and bioinformatics, we observed changes in AS patterns in response to light in Physcomitrella protonema. This finding indicates that AS is also abundant in non-vascular plants and that it can be regulated by light to alter the transcriptome for photomorphogenesis. We also observed immediate induction of IR when dark-grown cells exposed to the light (Figure 3). Such IR induction potentially generates unproductive transcripts or truncated proteins, thus resulting in the suppression of cellular processes. This process could be a safety procedure to temporarily attenuate cell growth for preventing light damage. In the later stage of light exposure, IR then becomes moderate and can promote vegetative growth under light. Expression of regulators in RNA splicing and light signaling is also 
modulated at the mRNA splicing step for splicing control and light-mediated gene expression.

To attenuate cell growth, an efficient way is to control key biological processes. Our data indicate that immediate IR induction by light occurs largely in transcripts of photosynthesis and ribosomal proteins (Figure 3). Both are important for energy and protein production in plant cells. Photosynthesis activity needs to be tightly controlled in response to light conditions. When gene transcription is not yet regulated by light signaling during the initial stage of light exposure, immediate induction of IR on photosynthesis gene transcripts could transiently decrease the production of photosynthetic proteins for function. Light repression of intron splicing on transcripts encoding ribosomal proteins could then globally inhibit translation in the cell or even play a role in specific translational control.

Besides metabolic processes, AS occurred in regulatory genes are also modulated by light. Light-induced intron splicing for SR protein gene transcripts suggest that although these splicing factors may not be primarily involved the immediate regulation of AS during light exposure, they could be important for subsequent splicing regulation in response to light conditions. Differential IR regulation under red and blue light for these genes further support that splicing factors may function differently under specific light conditions. We also observed significant amount of light signaling genes are alternatively spliced in response to light (Figure 5 and Table 2). AS of these key regulators could have a strong impact on photomorphogenic control for plants to increase transcriptome plasticity upon light exposure.

\section{Involvement of photoreceptors for splicing regulation}

In our study, two wavelengths of light were used to determine the light-dependent AS events. In the analysis of light-regulated IR, although the number of IR events responding to red and blue light were similar, events commonly regulated by both red and blue light are minor (Figure 1B). A large proportion of IR events were specifically regulated only by red or blue light. These observations support that red and blue light-dependent mechanisms may exist for regulating IR of different transcripts. Our hypothesis is further strengthened by the results of IR analysis in phytochrome-deficient and knockout mutants (Figure 8). Misregulation occurred in mutants for those light-responsive IR events reveal that phytochromes are primarily involved in the regulatory process. Because cryptogam phytochromes can also accumulate in the nucleus in a light-dependent manner [48], we therefore propose that red light-promoted splicing regulation in Physcomitrella is primarily mediated by light-activated, nuclear-localized phytochromes. Our data also indicate that phyB-type phytochromes play the dominant role in red light-regulated AS (Figure 8B), suggesting that wavelength-dependent mechanisms for splicing regulation are present in plants. Because the immediate, light-dependent AS regulation also occurs under the blue light condition, we believe blue-light photoreceptors are also required for the splicing regulation. Further analysis will be needed to support this hypothesis.

A similar pattern of IR induction and regulatory elements were found in both red and blue light-regulated IR events (Figure 7). A possible explanation is that red and blue light may affect the same splicing component(s) for regulation, but there are red and blue light-dependent mechanisms to determine transcript specificity of AS. One potential mechanism is through light-mediated chromatin modification as described below. Studies for the role of photoreceptors in AS regulation is undergoing.

\section{AS in plants possesses transcript specificity in response to environmental factors}

AS is commonly found in eukaryotic organisms and plays a major role in increasing overall transcriptome complexity and proteomic diversity. However, our data showed that light-regulated AS is predominant in transcripts involved in specific biological processes (Table 1). A question is how the splicing machinery differentiates AS transcripts. One possibility for transcript selection is the co-transcriptional control mediated by chromatin modification. Accumulating studies in AS regulation have provided new views of the co-transcriptional splicing mediated by epigenetic control [50,51]. Histone modification can affect AS by influencing the recruitment of splicing regulators to chromatin [52-54]. In plants, chromatin remodeling associated with light signaling is known for transcriptional regulation [13]. Photosynthesis genes have been shown as the targets of light-mediated chromatin modification during deetiolation [55-59]. Photoreceptor systems are possibly involved in light control for histone modifications [55]. In our analysis, AS of photosynthesis genes were also regulated in a light-dependent manner. We also found that AS occurs actively in the $\mathrm{PpHYH2}$ gene (Figure 6). Interestingly, the Arabidopsis HY5 and HYH loci revealed significant light-dependent histone modification [56]. These results reveal a possible link between lightdependent regulation of AS and chromatin modification in plants. Further investigation will be required to support this hypothesis.

\section{Sequence-specific control of light-responsive AS}

Splicing regulators are also potential candidates involved in transcript-specific AS in response to light. In the light-regulated IR regions, we identified a purine-rich, 
repetitive GAA motif in exonic regions that potentially function as a regulatory element on IR transcripts. The repetitive GAA motif was enriched in light-regulated IR regions, which suggests its involvement in splicing regulation under light. This purine-rich sequence with GAA repeats on pre-mRNA is a splicing enhancer in mammalian systems [60-62]. An AG-rich exonic cis element has been shown capable of promoting downstream donor site recognition in plants [63]. In Arabidopsis, the repetitive GAA sequence was proposed as an exonic splicing enhancer (ESE) [64] (Arabidopsis 2010 project, [65]). This purine-rich splicing enhancer is recognized by SR proteins in animals $[66,67]$. A recent study has also shown purine-rich GAAG repeats in the intronic region function as the binding site for SCL33 protein in Arabidopsis [68]. Plant SR proteins may recognize the purine-rich sequence to regulate intron splicing. In our study, the GAA motif we identified is present in the IR regions mostly induced by light, which suggests this motif either functions as a silencer or losses enhancer activity during light-mediated splicing regulation for specific transcripts.

The status of splicing regulators could be the determinant for splicing regulation. For example, subcellular localization of SR proteins can modulate the splicing activity, because nuclear localization of splicing regulators is dynamic [69]. Moreover, post-translational modification can also affect the activity of SR proteins. The arginine/serine (RS) domain of SR proteins is the prominent target for phosphorylation [70,71]. Likely, lightmediated phosphorylation/dephosphorylation of specific splicing regulators allows the splicing machinery to differentiate transcripts for AS and control splice site recognition. We propose that unknown splicing regulators are involved in regulating IR of specific transcripts in response to light. How the purine-rich cis element regulates IR in plants requires the identification and characterization of its trans binding factors.

\section{Evolution of light-mediated transcriptional and post-transcriptional regulation}

Mosses are ancient non-vascular plants diverged early in land plant evolution. Therefore, the evolutionary position of mosses in the intermediate group of green lineage reveals its importance in the study of conserved biological processes for photosynthetic organisms. In the previous study, we have observed that light-mediated transcriptional regulation is conserved at least in part among land plants. The occurrence of AS in the moss is also comparable to that in higher plants. Nevertheless, the finding of abundant AS events in moss protonema cells strongly supports that AS patterns could have diversified already during land colonization of aquatic photosynthetic organisms, and retained in higher plants.

\section{Conclusions}

In this study, we used RNA-seq to study light-regulated gene expression at the post-transcriptional level in Physcomitrella. We have provided the first genome-wide analysis to show that alternative splicing is regulated by light in plants. Evidence from this study suggests that the mRNA splicing step can be rapidly modulated by light to control AS of transcripts involved in specific functions. Such regulation is important for light responses in plants. We have also identified a regulatory cis element involved in light-mediated splicing regulation. This result may allow identifying the potential RNAbinding proteins or splicing regulators involved in the light-mediated AS regulation. We also found that IR was rapidly induced by light but misregulated in moss mutants defective in red light sensing phytochromes, suggesting that photoreceptors primarily participate in regulating AS in plants. Our study is the first to report a transcriptomewide analysis for AS in non-vascular plants. The results demonstrate that patterns of AS are evolutionarily conserved in land plants. Although the current approach is unable to reveal the mechanism of light-regulated AS, it provides many new directions to pursue. We hope that further investigation on the regulatory mechanism of mRNA splicing can shed light on studies of gene regulation in plants in response to environmental factors.

\section{Materials and methods}

\section{Plant materials and growth conditions}

Protonemata of Physcomitrella patens subsp. patens were grown on solid Knop's medium or solid BCDAT medium. Spores were germinated on solid Knop's medium supplemented with $10 \mathrm{mM} \mathrm{CaCl}_{2}$ and overlaid with cellophane for approximately 14 to 16 days. Plants were cultured at $25^{\circ} \mathrm{C}$ under continuous white light (80-100 $\mu \mathrm{mol} \mathrm{m}^{-2} \mathrm{~s}^{-1}$ ).

\section{Light treatment, RNA isolation, and mRNA sequencing}

The 10-day-old protonemata of the WT were grown in the dark for 3 days followed by 1 - and 4-h red-light treatment $\left(660 \mathrm{~nm}\right.$ LED, $5 \mu \mathrm{mol} \mathrm{m} \mathrm{s}^{-1}$ ) at $25^{\circ} \mathrm{C}$ as previously reported [34]. For blue-light treatment, a light intensity similar to that in a previous study was used (472 $\mathrm{nm} \mathrm{LED,} 17 \mu \mathrm{mol} \mathrm{m}{ }^{-2} \mathrm{~s}^{-1}$ ) [72]. Dark-grown protonema cells were collected as the dark control. RNA isolation and cDNA library preparation were performed as described previously [34]. Sequencing was performed on the Hiseq 2000 at Yourgene Bioscience, Taiwan. On average, 39 million 100-nt paired-end reads for each sample were obtained.

\section{Read mapping to the reference genome}

Sequence reads were mapped to the Physcomitrella patens genome V1.6 ([73], data can also be found in JGI, 
[74]) by use of the BLAT program [35,75]. Reads per kilobase of exon model per million mapped reads (RPKMs) computation involved use of the RACKJ package ([76]) with a similar algorithm as described previously [36].

\section{Prediction of AS}

RACKJ was used to compute the following read counts (mapping by BLAT) and separated into several tables: (1) for every exon; (2) for every intron; (3) for every exon-pair that were mapped by splice reads; and (4) for every exon-pair plus junction shifts that were supported by spliced reads. AS events were then computed accordingly. The fourth table records potential AltA/AltD events. From the aforementioned tables, perl scripts in RACKJ package ([76]) were used for detecting AS (IR, ES, and AltA/AltD) events for red- and blue-light samples [37]. Chi-square values for goodness-of-fit were computed by comparing read counts supporting an AS event (that is, intronic read counts, exon-skipped read counts, and alternative donor/acceptor read counts) and read counts not supporting the AS event (for example, read counts of corresponding gene exons, read counts of neighboring exons, read counts involving a skipped exon, and read counts of the same exon-pair but not of the same AltA/AltD events). In so doing, we then computed corresponding $P$ values by chi-square distribution using Microsoft Excel (Microsoft, Redmond, WA, USA).

\section{Gene ontology analysis}

Functional enrichment analysis involved the Gene Ontology Browsing Utility (GOBU) with MultiView ([77]) [78]. Physcomitrella gene sequences were first subjected to the Bio301 system to generate GO annotations for all transcripts [79]. The Bio301 system predicts functions by blasting against UniProt and RefSeq databases and by InterProt domain prediction to generate the reference GO annotation set. $P$ values for over-represented GO terms were computed by comparing the differentially regulated gene set to the reference $\mathrm{GO}$ annotation set using the 'elim' method. The elim method is a Java implementation of the TopGO 'elim' algorithm [80]. For generating heat maps, relative IR and gene expression level were plotted by use of Java Treeview [81].

\section{Analysis of cis regulatory elements}

Motif search involved the MEME suite [44]. An extra run of MAST, a member program of MEME, with the option -hit_list was performed for searching motif sites on promoter or AS regions. Note that every predicted motif site in the MAST output is associated with a $P$ value for similarity. To compute the preference of a given motif under a certain MAST $P$ value threshold, Fisher's exact test was performed with the following four numbers: (1) light-regulated promoter or AS regions hit (that is, input sequences with at least one motif occurrence); (2) randomly selected promoter or AS regions hit; (3) light-regulated promoter or AS regions not hit; and (4) randomly selected promoter or AS regions not hit. A motif that is preferentially present in the lightregulated promoter or AS regions would show a significant $P$ value.

\section{Quantitative RT-PCR analysis}

The cDNA synthesis was performed with $4.5 \mu \mathrm{g}$ of total RNA, an oligo(dT), and SuperScript III RT kit (Invitrogen, USA). qRT-PCR analysis involved the 7500 Real Time PCR System (Applied Biosystems, USA) with the Power SYBR Green PCR Master Mix (Applied Biosystems, USA). Primers designed by Primer Express Software v3.0 (Applied Biosystems, USA) are in Additional file 7. qRT-PCR reactions were in triplicate. PpACT2 (Pp1s198_157V6) was used as an internal control for normalization in qRT-PCR.

\section{High-resolution RT-PCR}

High-resolution RT-PCR was performed as described previously with minor modification [43]. PCR involved AS-specific primers (Additional file 7). One strand of the primer pair was labeled with the fluorescent dye 5 -carboxyfluorescein (5'-FAM). RT-PCR product $(1 \mu \mathrm{L})$ was diluted into $10 \mu \mathrm{L}$ mixture of $\mathrm{Hi}$-Di formamide and GeneScan 600 LIZ internal size standard (90:1 ratio) (Applied Biosystems, USA). DNA fragments were separated on an ABI3730 DNA Analyzer (Applied Biosystems, USA) with three technical repeats and then analyzed by use of Peak Scanner v1.0. RT-PCR products from corresponding AS isoforms were identified with expected sizes. The percentage of each AS isoform was calculated as the fluorescent peak areas of AS transcripts divided by the sum of all detected transcripts. In comparing results from three samples by ANOVA, $P$ values were generated.

\section{Data deposition}

RNA-seq data from this publication have been submitted to the National Center for Biotechnology InformationSequence Read Archive database (SRA, [82]) and assigned the identifier SRX252526.

\section{Additional files}

Additional file 1: Contains Supplementary Figures S1 to S5 and Supplementary Tables S1 to S3.

Additional file 2: Tables listing all and statistically significant IR, AltDA, AltA, AltD, and ES events found in red-light data.

Additional file 3: Tables listing all and statistically significant IR, AltDA, AltA, AltD, and ES events found in blue-light data. 
Additional file 4: Tables listing splicing-related genes found in statistically significant IR events of red-light data.

Additional file 5: Tables listing splicing-related genes found in statistically significant IR events of blue-light data.

Additional file 6: Tables listing light signaling genes found in statistically significant IR events of red- and blue-light data.

Additional file 7: Table listing all primers used in this study.

\section{Competing interests}

The authors declare that they have no competing interests.

\section{Authors' contributions}

S-LT designed the research. H-PW, Y-SS, Y-RC, H-CC, C-CW, and W-DL performed the research. H-PW, Y-SS, and S-LT analyzed the data. S-LT wrote the paper. All authors read and approved the final manuscript.

\section{Acknowledgements}

We thank Shu-Hsing Wu, Chiung-swey Chang, and Laura Smales for critically reading the manuscript, and Mei-Jane Fang in the DNA Analysis Core Laboratory of the Institute of Plant and Microbial Biology, Academia Sinica for technical assistance. We also thank the National Center for Genome Medicine at Academia Sinica, Taiwan, for genotyping/technical support. The Center was supported by grants from the National Core Facility Program for Biotechnology of National Science Council, Taiwan. This project was supported by a Career Development Award (to SLT), Academia Sinica.

Received: 17 July 2013 Accepted: 07 January 2014

Published: 07 January 2014

\section{References}

1. Maniatis T: Mechanisms of alternative pre-mRNA splicing. Science 1991, 251:33-34.

2. Reddy AS: Alternative splicing of pre-messenger RNAs in plants in the genomic era. Annu Rev Plant Biol 2007, 58:267-294.

3. Black DL: Mechanisms of alternative pre-messenger RNA splicing. Annu Rev Biochem 2003, 72:291-336.

4. Syed NH, Kalyna M, Marquez Y, Barta A, Brown JWS: Alternative splicing in plants - coming of age. Trends Plant Sci 2012, 17:616-623.

5. Filichkin SA, Priest HD, Givan SA, Shen R, Bryant DW, Fox SE, Wong W-K, Mockler TC: Genome-wide mapping of alternative splicing in Arabidopsis thaliana. Genome Res 2010, 20:45-58.

6. Marquez Y, Brown JWS, Simpson C, Barta A, Kalyna M: Transcriptome survey reveals increased complexity of the alternative splicing landscape in Arabidopsis. Genome Res 2012, 22:1184-1195.

7. Zhang G, Guo G, Hu X, Zhang Y, Li Q, Li R, Zhuang R, Lu Z, He Z, Fang X, Chen L, Tian W, Tao Y, Kristiansen K, Zhang X, Li S, Yang H, Wang J: Deep RNA sequencing at single base-pair resolution reveals high complexity of the rice transcriptome. Genome Res 2010, 20:646-654.

8. Chang Y-F, Imam JS, Wilkinson MF: The nonsense-mediated decay RNA surveillance pathway. Ann Rev Biochem 2007, 76:51-74.

9. Kalyna M, Simpson CG, Syed NH, Lewandowska D, Marquez Y, Kusenda B, Marshall J, Fuller J, Cardle L, McNicol J, Dinh HQ, Barta A, Brown JW: Alternative splicing and nonsense-mediated decay modulate expression of important regulatory genes in Arabidopsis. Nucleic Acids Res 2012, 40:2454-2469

10. Briggs WR: Spudich JA (eds): Handbook of Photosensory Receptors. Wiley-VCH: Weinheim; 2005

11. Kami C, Lorrain S, Hornitschek P, Fankhauser C: Light-regulated plant growth and development. In Current Topics in Developmental Biology, Volume 91. Edited by Marja CPT. Waltham, MA: Academic Press; 2010:29-66.

12. Huq E, Quail PH: Phytochrome signaling. In Handbook of Photosensory Receptors. Edited by Briggs WR, Spudich JA. Weinheim: Wiley-VCH; 2005:151-170

13. Fisher AJ, Franklin KA: Chromatin remodelling in plant light signalling. Physiol Plant 2011, 142:305-313.

14. Li J, Terzaghi W, Deng XW: Genomic basis for light control of plant development. Protein Cell 2012, 3:106-116.
15. Schroeder DF, Gahrtz M, Maxwell BB, Cook RK, Kan JM, Alonso JM, Ecker JR, Chory J: De-etiolated 1 and damaged DNA binding protein 1 interact to regulate Arabidopsis photomorphogenesis. Curr Biol 2002, 12:1462-1472.

16. Casal JJ, Yanovsky MJ: Regulation of gene expression by light. Int J Dev Biol 2005, 49:501-511.

17. Jenkins GI: Signal transduction in responses to UV-B radiation. Annu Rev Plant Biol 2009, 60:407-431.

18. Chen M, Chory J: Phytochrome signaling mechanisms and the control of plant development. Trends Cell Biol 2011, 21:664-671.

19. Leivar P, Quail PH: PIFs: pivotal components in a cellular signaling hub. Trends Plant Sci 2011, 16:19-28.

20. Petracek ME, Dickey LF, Huber SC, Thompson WF: Light-regulated changes in abundance and polyribosome association of ferredoxin mRNA are dependent on photosynthesis. Plant Cell 1997, 9:2291-2300.

21. Helliwell CA, Webster Cl, Gray JC: Light-regulated expression of the pea plastocyanin gene is mediated by elements within the transcribed region of the gene. Plant J 1997, 12:499-506.

22. Dickey LF, Petracek ME, Nguyen TT, Hansen ER, Thompson WF: Light regulation of Fed-1 mRNA requires an element in the $5^{\prime}$ untranslated region and correlates with differential polyribosome association. Plant Cell 1998, 10:475-484.

23. McKim SM, Durnford DG: Translational regulation of light-harvesting complex expression during photoacclimation to high-light in Chlamydomonas reinhardtii. Plant Physiol Biochem 2006, 44:857-865.

24. Liu M-J, Wu S-H, Chen H-M, Wu S-H: Widespread translational control contributes to the regulation of Arabidopsis photomorphogenesis. Mol Syst Biol 2012, 8:566.

25. Lau OS, Deng XW: The photomorphogenic repressors COP1 and DET1: 20 years later. Trends Plant Sci 2012, 17:584-593.

26. Wei N, Deng XW: The COP9 signalsome. Ann Rev Cell Dev Biol 2003, 19:261-286.

27. Mastrangelo AM, Marone D, Laidò G, De Leonardis AM, De Vita P: Alternative splicing: Enhancing ability to cope with stress via transcriptome plasticity. Plant Sci 2012, 185-186:40-49.

28. Mano S, Hayashi M, Nishimura M: A leaf-peroxisomal protein, hydroxypyruvate reductase, is produced by light-regulated alternative splicing. Cell Biochem Biophys 2000, 32:147-154.

29. Yoshimura K, Mori T, Yokoyama K, Koike Y, Tanabe N, Sato N, Takahashi H, Maruta T, Shigeoka S: Identification of alternative splicing events regulated by an Arabidopsis serine/arginine-like protein, atSR45a, in response to high-light stress using a tiling array. Plant Cell Physiol 2011 , 52:1786-1805

30. Seo PJ, Park MJ, Lim MH, Kim SG, Lee M, Baldwin IT, Park CM: A self-regulatory circuit of CIRCADIAN CLOCK-ASSOCIATED1 underlies the circadian clock regulation of temperature responses in Arabidopsis. Plant Cell 2012, 24:2427-2442

31. Penfield $\mathrm{S}$, Josse $E M$, Halliday $\mathrm{KJ}$ : A role for an alternative splice variant of PIF6 in the control of Arabidopsis primary seed dormancy. Plant Mol Biol 2010, 73:89-95.

32. Zhou DX, Kim YJ, Li YF, Carol P. Mache R: COP1b, an isoform of COP1 generated by alternative splicing, has a negative effect on COP1 function in regulating light-dependent seedling development in Arabidopsis. Mol Gen Genet 1998, 257:387-391.

33. Shikata H, Shibata M, Ushijima T, Nakashima M, Kong SG, Matsuoka K, Lin C, Matsushita T: The RS domain of Arabidopsis splicing factor RRC1 is required for phytochrome B signal transduction. Plant J 2012, 70:727-738.

34. Chen Y-R, Su Y-s, Tu S-L: Distinct phytochrome actions in nonvascular plants revealed by targeted inactivation of phytobilin biosynthesis. Proc Natl Acad Sci USA 2012, 109:8310-8315.

35. Zimmer AD, Lang D, Buchta K, Rombauts S, Nishiyama T, Hasebe M, Van de Peer Y, Rensing SA, Reski R: Reannotation and extended community resources for the genome of the non-seed plant Physcomitrella patens provide insights into the evolution of plant gene structures and functions. BMC Genomics 2013, 14:498.

36. Mortazavi A, Williams BA, McCue K, Schaeffer L, Wold B: Mapping and quantifying mammalian transcriptomes by RNA-Seq. Nat Meth 2008 5:621-628

37. Li W, Lin W-D, Ray P, Lan P, Schmidt W: Genome-wide detection of condition-sensitive alternative splicing in Arabidopsis roots. Plant Physiol 2013, 162:1750-1763.

38. Lan P, Li W, Lin W-D, Santi S, Schmidt W: Mapping gene activity of Arabidopsis root hairs. Genome Biol 2013, 14:R67. 
39. Wang BB, Brendel $V$ : The ASRG database: identification and survey of Arabidopsis thaliana genes involved in pre-mRNA splicing. Genome Biol 2004, 5:R102.

40. Reddy ASN, Shad Ali G: Plant serine/arginine-rich proteins: roles in precursor messenger RNA splicing, plant development, and stress responses. Wiley Interdisciplinary Reviews: RNA 2011, 2:875-889.

41. Holm M, Ma L-G, Qu L-J, Deng X-W: Two interacting bZIP proteins are direct targets of COP1-mediated control of light-dependent gene expression in Arabidopsis. Gene Dev 2002, 16:1247-1259.

42. Morris DR, Geballe AP: Upstream open reading frames as regulators of mRNA translation. Mol Cell Biol 2000, 20:8635-8642.

43. Simpson CG, Fuller J, Maronova M, Kalyna M, Davidson D, McNicol J, Barta A, Brown JW: Monitoring changes in alternative precursor messenger RNA splicing in multiple gene transcripts. Plant J 2008, 53:1035-1048.

44. Bailey TL, Boden M, Buske FA, Frith M, Grant CE, Clementi L, Ren J, Li WW, Noble WS: MEME Suite: tools for motif discovery and searching. Nucl Acids Res 2009, 37:W202-W208.

45. Rensing SA, Lang D, Zimmer AD, Terry A, Salamov A, Shapiro H, Nishiyama T, Perroud P-F, Lindquist EA, Kamisugi Y, Tanahashi T, Sakakibara K, Fujita T, Oishi K, Shin-I T, Kuroki Y, Toyoda A, Suzuki Y, Hashimoto S-i, Yamaguchi K, Sugano S, Kohara Y, Fujiyama A, Anterola A, Aoki S, Ashton N, Barbazuk WB, Barker E, Bennetzen JL, Blankenship R: The Physcomitrella genome reveals evolutionary insights into the conquest of land by plants. Science 2008, 319:64-69.

46. Mittmann F, Brucker G, Zeidler M, Repp A, Abts T, Hartmann E, Hughes J: Targeted knockout in Physcomitrella reveals direct actions of phytochrome in the cytoplasm. Proc Natl Acad Sci USA 2004, 101:13939-13944.

47. Uenaka H, Kadota A: Functional analyses of the Physcomitrella patens phytochromes in regulating chloroplast avoidance movement. Plant J 2007, 51:1050-1061.

48. Possart A, Hiltbrunner A: An evolutionarily conserved signaling mechanism mediates far-red light responses in land plants. Plant Cell 2013, 25:102-114

49. Mittmann F, Dienstbach S, Weisert A, Forreiter C: Analysis of the phytochrome gene family in Ceratodon purpureus by gene targeting reveals the primary phytochrome responsible for photo- and polarotropism. Planta 2009, 230:27-37.

50. Luco RF, Allo M, Schor IE, Kornblihtt AR, Misteli T: Epigenetics in alternative pre-mRNA slicing. Cell 2011, 144:16-26.

51. Schwartz S, Ast G: Chromatin density and splicing destiny: on the cross-talk between chromatin structure and splicing. EMBO J 2010 29:1629-1636

52. Luco RF, Pan Q, Tominaga K, Blencowe BJ, Pereira-Smith OM, Misteli T: Regulation of alternative splicing by histone modifications. Science 2010, 327:996-1000

53. Pradeepa MM, Sutherland HG, Ule J, Grimes GR, Bickmore WA: Psip1/Ledg p52 binds methylated histone H3K36 and splicing factors and contributes to the regulation of alternative splicing. PLOS Genet 2012, 8:e1002717.

54. Moehle EA, Ryan CJ, Krogan NJ, Kress TL, Guthrie C: The yeast SR-like protein Npl3 links chromatin modification to mRNA processing. PLOS Genet 2012, 8:29.

55. Guo L, Zhou J, Elling AA, Charron JB, Deng XW: Histone modifications and expression of light-regulated genes in Arabidopsis are cooperatively influenced by changing light conditions. Plant Physiol 2008, 147:2070-2083.

56. Charron JB, He H, Elling AA, Deng XW: Dynamic landscapes of four histone modifications during deetiolation in Arabidopsis. Plant Cell 2009, 21:3732-3748

57. Chua YL, Brown AP, Gray JC: Targeted histone acetylation and altered nuclease accessibility over short regions of the pea plastocyanin gene. Plant Cell 2001, 13:599-612.

58. Chua YL, Watson LA, Gray JC: The transcriptional enhancer of the pea plastocyanin gene associates with the nuclear matrix and regulates gene expression through histone acetylation. Plant Cell 2003, 15:1468-1479.

59. Benhamed M, Bertrand C, Servet C, Zhou DX: Arabidopsis GCN5, HD1, and TAF1/HAF2 interact to regulate histone acetylation required for light-responsive gene expression. Plant Cell 2006, 18:2893-2903.

60. Staffa A, Cochrane A: Identification of positive and negative splicing regulatory elements within the terminal tat-rev exon of human immunodeficiency virus type 1. Mol Cell Biol 1995, 15:4597-4605.
61. Yeakley JM, Hedjran F, Morfin JP, Merillat N, Rosenfeld MG, Emeson RB: Control of calcitonin/calcitonin gene-related peptide pre-mRNA processing by constitutive intron and exon elements. Mol Cell Biol 1993, 13:5999-6011.

62. Tanaka K, Watakabe A, Shimura Y: Polypurine sequences within a downstream exon function as a splicing enhancer. Mol Cell Biol 1994, 14:1347-1354

63. McCullough AJ, Schuler MA: Intronic and exonic sequences modulate 5' splice site selection in plant nuclei. Nucl Acids Res 1997, 25:1071-1077.

64. Pertea M, Mount SM, Salzberg SL: A computational survey of candidate exonic splicing enhancer motifs in the model plant Arabidopsis thaliana. BMC Bioinformatics 2007, 8:159.

65. Arabidopsis 2010 project to characterize pre-mRNA splicing signals [http://www.life.umd.edu/labs/mount/2010-splicing/ESEs.html]

66. Yeakley JM, Morfin JP, Rosenfeld MG, Fu XD: A complex of nuclear proteins mediates SR protein binding to a purine-rich splicing enhancer. Proc Natl Acad Sci USA 1996, 93:7582-7587.

67. Ramchatesingh J, Zahler AM, Neugebauer KM, Roth MB, Cooper TA: A subset of SR proteins activates splicing of the cardiac troponin $T$ alternative exon by direct interactions with an exonic enhancer. Mol Cell Biol 1995, 15:4898-4907.

68. Thomas J, Palusa SG, Prasad KV, Ali GS, Surabhi GK, Ben-Hur A, Abdel-Ghany SE, Reddy AS: Identification of an intronic splicing regulatory element involved in auto-regulation of alternative splicing of SCL33 pre-mRNA. Plant J 2012, 72:935-946.

69. Will CL, Urlaub H, Achsel T, Gentzel M, Wilm M, Luhrmann R: Characterization of novel SF3b and 17S U2 snRNP proteins, including a human Prp5p homologue and an SF3b DEAD-box protein. EMBO J 2002, 21:4978-4988

70. Manley JL, Tacke R: SR proteins and splicing control. Gene Dev 1996, 10:1569-1579.

71. Graveley BR: Sorting out the complexity of SR protein functions. RNA 2000, 6:1197-1211

72. Imaizumi T, Kadota A, Hasebe M, Wada M: Cryptochrome light signals control development to suppress auxin sensitivity in the moss Physcomitrella patens. Plant Cell 2002, 14:373-386.

73. Cosmoss database. http://cosmoss.org/

74. Phytozome. http://www.phytozome.net/physcomitrella.php.

75. Kent WJ: BLAT-The BLAST-like alignment tool. Genome Res 2002, 12:656-664

76. Read analysis \& comparison kit in Java. http://rackj.sourceforge.net/.

77. Gene ontology browsing utility. http://gobu.openfoundry.org/.

78. Lin W-D, Chen Y-C, Ho J-M, Hsiao C-D: GOBU: toward an integration interface for biological objects. J Inf Sci Eng 2006, 22:19-29.

79. Chen Y-C, Chen Y-C, Lin W-D, Hsiao C-D, Chiu H-W, Ho J-M: Bio301: a web-based EST annotation pipeline that facilitates functional comparison studies. ISRN Bioinformatics 2012, 2012:139842

80. Alexa A, Rahnenfuhrer J, Lengauer T: Improved scoring of functional groups from gene expression data by decorrelating GO graph structure. Bioinformatics 2006, 22:1600-1607.

81. Saldanha AJ: Java Treeview-extensible visualization of microarray data. Bioinformatics 2004, 20:3246-3248.

82. National Center for Biotechnology Information-Sequence Read Archive database [http://www.ncbi.nlm.nih.gov/sra]

doi:10.1186/gb-2014-15-1-r10

Cite this article as: Wu et al:: Genome-wide analysis of light-regulated alternative splicing mediated by photoreceptors in Physcomitrella patens. Genome Biology 2014 15:R10. 